\title{
Activity limitations and factors influencing functional outcome of patients with stroke following rehabilitation at a specialised facility in the Western Cape
}

\author{
*Joseph C, Rhoda A \\ Department of Physiotherapy, University of the Western Cape, Cape Town, South Africa
}

\begin{abstract}
Background: Determining the functional abilities and factors influencing outcome of patients with stroke following rehabilitation are essential for the planning of future interventions and services in order to optimise recovery.

Objectives: To determine the activity limitations and factors influencing functional outcome of patients with stroke managed at a specialised rehabilitation centre.

Methodology: A longitudinal study design was used to determine the functional outcomes of patients admitted to the centre on admission and discharge. A data gathering sheet was developed to collect information pertaining to the demographicand medical profile and process of rehabilitation, whereas the Barthel Index was used to collect data relating to functional abilities. For analysis, descriptive statistics as well as inferential statistics (Student $t$ test) were utilised to determine the paired differences. Six prognostic factors influencing functional outcome were selected and tested using linear (bivariate) regression. Results: The mean Barthel Index scores on admission and at discharge were 58.85 and 81.59 respectively. A significant improvement was noted in the execution of functional task of patients with stroke $(p<0.01)$ between the data collection points. Despite the significant overall improvement, results show a high prevalence of dependence with walking and stair climbing at discharge. Only functional ability on admission $(\mathrm{r}=0.49)$ predicted a favourable functional outcome at discharge. Conclusion: This study highlights the limitations of younger stroke survivors and the need for continued rehabilitation following in-patient care. It further underscores the administration of a functional rating scale on admission in order to aggressively manage activity limitations.
\end{abstract}

Key words: stroke, activity limitations, factors influencing outcome, ICF, rehabilitation

African Health Sciences 2013; 13(3): 646 - 654 http://dx.doi.org/10.4314/ahs.v13i3.18

\section{Introduction}

The World Health Organisation (WHO) (2001) identified stroke as one of the leading causes of death and morbidity worldwide ${ }^{1}$, which appears to affect 243-300 per 100000 of the South African population ${ }^{2}$. Not only does stroke result in longterm disability, but it further has the potential of impacting the patient, family and state as well as leading to dependency on others for assistance with everyday activities ${ }^{2-3}$. Literature clearly underscores that the functional independence of persons with stroke is significantly lower than that of the rest of

*Corresponding author
Conran Joseph
Karolinska Institutet
Department of Neurobiology, Care Sciences and
Society
Division of Physiotherapy
Zanderhuset, Alfred Nobels Allé 23
SE-14183 Huddinge
Sweden
Email: conran.joseph@ki.se

the population, which further highlights the disabling consequences of stroke 4 . Thus, in order to respond to the healthcare and rehabilitation needs of persons with stroke, a recent study undertaken in South Africa investigated the influence of specialised in-patient rehabilitation on the functional outcomes and also to identify predictors of functional outcomes. This investigation is important in order to better focus future interventions, services and policies.

A stroke does not only affect patients on a pathophysiological level, but extend beyond the medical characteristics altered by the health condition. The influence of a stroke on the functioning of patients can be understood using the International Classification of Functioning, Disability and Health (ICF) as conceptual model. This conceptual model or classification not only acknowledges the dysfunction or deviation of bodily function and structure in the form of impairments, but encapsulates the burden of activity limitations and participation restrictions experienced by the patients in his or her unique architectural-, social- and 
attitudinal environment ${ }^{1}$. Furthermore, this framework allows the underpinning of key novel contributions of the contextual factors on the functioning of the patient, which is essential for context-specific rehabilitation interventions ${ }^{5}$.

As the understanding of disability evolved over time, so has the term rehabilitation, which is loosely defined as the strategy to address disability through discipline-specific interventions and the collaborate efforts of different governmental sectors $^{6}$. Within the last three decades there have been a divergence of rehabilitation from its approach being grounded within the medical model of disability to the so-called bio-psycho-social model (ICF), thus rehabilitation towards addressing outcomes important to the service user (which are often related to functional abilities and occupational duties) have become pivotal. These functional abilities have become the reference framework against which meaningful outcomes for the patients are measured, and the impetus for the provision of rehabilitation services along the continuum of care, and not just intermittently on an in-patient basis ${ }^{1}$. The literature suggests that community-dwelling individuals with stroke usually experience difficulties with self care activities and mobility tasks ${ }^{7-9}$. The latter has also been found to predict community reintegration and return to work $^{10}$. As these limitations continue to persist along the chronic stages of the stroke, it has become important to quantify the number of patients presenting with similar limitation in order to provide a coherent argument against the content-, intensityand contextually appropriateness of the rehabilitation interventions.

In order to systematically and objectively measure the functional gains of patients with stroke, standardised functional outcome measures were developed ${ }^{11}$, and many argue that the tools classified as the "gold standard" for a particular group of people have the potential of underpinning the efficacy and role of rehabilitation. Comprehensive, patientspecific rehabilitation practices are currently the desired approach for addressing patient problems and for optimising functioning in all spheres of human functioning. To achieve the desired level of functioning, factors predictive of outcome should be determined and counteracted by appropriate services or processes ${ }^{6,12}$. Studies investigating the factors influencing outcome reported that personal factors such as age, gender, severity and aetiology of injury may provide an indication of prognosis for recovery ${ }^{13}$. Apart from personal factors influencing outcome, studies reported the use of certain protocols and services to account for much of the variance in functional outcome. Since the ICF framework acknowledges the context-specifics of an individual and the execution of functional tasks within a context, it is imperative to have insight into the factors predictive of functional outcome of individuals between different settings and geographical areas in order to inform the rehabilitation process for a particular group of individuals.

Stroke onset in the Western Cape, South Africa, takes place at a much younger age than persons in the developed world ${ }^{2,7}$, which greatly underscores the longer time these individuals have to live with the health condition. In addition, the likelihood of persons with stroke receiving lifelongor community-based rehabilitation along with inpatient rehabilitation is unlikely due to the highly pressurised healthcare system in South Africa. In the light of the challenges to the provision of rehabilitation services along a continuum, this study will provide valuable information on the prevalence of limitations of certain tasks essential for independent living and will further shed light on the need to investigate the content of the current rehabilitation interventions utilised to address tasks with high levels of limitations. In this way the content of rehabilitation could become more directive in future. Given these theoretical arguments, the baseline study not only sets out to determine the activity limitations, but also investigates the factors influencing functional outcome of patients with a stroke following specialised in-patient rehabilitation.

\section{Methods}

\section{Setting, population and sampling}

This study was conducted at a specialised rehabilitation centre in the Western Cape, South Africa, which adopts an outcome-based approach to patient management. This centre manages patients with various diagnosed neurological conditions, with the majority of patients surviving a stroke or spinal cord injury. A longitudinal, observational study design was used to collect data pertaining to the functional outcomes of patients with stroke between admission and discharge. This design allows the researcher to describe the situation as it appears and without influencing the structure and process of care in any way. The document review methodology collated demographic-, medical- and impairment data, process of rehabilitation data, as well as collateral 
information related to the rehabilitation outcome levels achieved by patients on admission and at discharge. The study population consisted of consecutive patients presenting with stroke for a three month period (November 1, 2010-January 31, 2011). This time frame allowed for a sufficient sample size in order to study the six prognostic factors that were identified from the literature, which could predict functional outcome of patients with stroke. The inclusion criteria were all eligible participants aged 18 years and above presenting with stroke, those who consent in writing to participate and patients' proficient in at least one of the languages spoken by the people within the catchment area, which were English, Afrikaans or Isi-Xhosa. Those who were excluded either presented with a mental disability as a co-illness or who could not follow a three-step command test due to aphasia. The exclusion of patient with aphasia, using the three-step command test, is justified by the fact that relevant data on functional outcomes were collected via interview, which could have led to bias in responses.

\section{Instruments and data collection}

The Barthel Index, which presents with sound validity, reliability and responsiveness to change was utilised to measure the severity of functional limitations. The rating scale (presented with intervals of 5 points) of this tool allows for the measurement of the level of independence in functional activities, which included items related to basic mobility, self-care activities and functions of bladder and bowel continence. The score ranges from 0-100. A score lower than 50 indicates maximal dependence and above 75 represents minimal dependence. Thus, a higher BI score indicates better functioning ${ }^{14-15}$. The functional abilities of patients were subjectively performancebased gathered during interview, with the outcome measure presenting with substantial reliability when used in this format. The data collecting points were within two working days post admission and within the last two days prior to discharge.

The demographic-, medical data as well as the information relating to the process of rehabilitation and rehabilitation outcome were collated from the medical record of the patients at discharge. The items for the demographic- and medical profile included; age, gender, marital status, diagnosis, laterality of stroke. Certain items were also related to impairments following the stroke. Given the complexity of determining the extent of the impairment, cognitive function and abnormal tone were captured as either "impaired", "not impaired" or "undocumented". The documentation of the impairments was only retrieved by reviewing the doctors-, physiotherapist- or occupational therapists assessment- notes on admission. The items related to the process of rehabilitation and rehabilitation outcome levels were limited to the length of hospital stay (LOHS), discharge destination, health professionals involved in the management of the patients and outcome levels achieved on admission and at discharge. The use of rehabilitation outcome levels as a measure of outcome and prediction is unique to the institution. These levels represent categories or groupings of patient problems along the disease progress and provide a framework of how health care should be delivered and directed towards the specific health problems.

The rehabilitation outcome levels which were determined by the entire interdisciplinary team were recorded, using the medical records, on admission and at discharge and was thus used as a collateral to the interpretation of the functional outcomes as derived from the BI. Lastly, the six prognostic factors were identified from the data elements that were collected from the medical records and that is in accordance with literature based on the ICF and the service delivery model. The data gathering sheet was validated for content by two experts in the field of rehabilitation and was also found to be reliable when reviewed by two raters, with Intraclass Correlation Coefficient (ICC) scores ranging between 0.74-1.00 for the completed sheet.

\section{Ethical considerations}

All ethical principles related to research on human subjects, as derived from the Declaration of Helsinki, were adhered to throughout the data collection period and writing for publication. Permission to conduct the study and ethical approval was also obtained from all relevant authorities including the University's Senate Research Committee (Project registration number: 10/3/24).

\section{Statistical methods}

All data elements were coded and captured twice in Windows Excel 2007, and imported to SAS for analysis. Descriptive statistics were used for data elements related to the demographic and medical variables, rehabilitation outcome levels as well as individual items reflected on the standardised outcome measures. Results were presented as 
frequencies, means, standard deviations and percentages. The Shapiro Wilk W test was used to determine the distributed of data elements. Based on the outcome of the Shapiro Wilk test, which indicated the normal distribution of data, the Student $\mathrm{t}$ - test was used to determine the significant difference in functional outcome of patients between admission and discharge. For the measurement of significant difference in functional outcome, a confidence interval of $95 \%$ was chosen as an acceptable level ${ }^{16}$. For the study of predictors of functional outcome, a bivariate linear regression model was used. Due to the abnormality of data elements, non-parametric tests were done. For dichotomous predictors a Wilcoxon Rank Sum test was used, whereas the Spearman's rank order correlation co-efficient was used for the continuous independent variables ${ }^{16}$.

\section{Results}

Patients with stroke accounted for $38 \%$ of the total admissions to the centre for the period of November 01, 2010 to January 31, 2011. Of the 82 patients, one patient was under 18 years of age, two declined consent, three subjects presented with missing data and the remaining seven presented with co-morbidities and receptive aphasia. Thus, the sample meeting the inclusion criteria was 67 patients with stroke. The mean age and standard deviation (s.d.) of patients with stroke was 52.95 and 12.19 respectively, and with an age range of 23 to 79 years of age. The median age of 53 years corresponds closely with the mean age, with the mode of 44 years, which is significantly lower than the mean and median age. Most of the patients with stroke (54\%) were married at the time of the diagnosis of the disease and of the male gender $(52 \%)$. On examination, the laterality of the brain lesion was almost similar for either side, but with slightly more left side lesions $(50.75 \%)$.

\section{Process of rehabilitation}

All patients with stroke were managed by the nurse, with most of them managed by the doctor, physiotherapist and occupational therapist. The mean length of hospital stay was 51.62 days, with the majority of patients discharged to their long-term residence. Following in-patient rehabilitation only $20 \%$ of the sample received follow-up medical or rehabilitation services at either the institution's outpatient department or another health care facility. The majority of the patients were admitted with a health status corresponding to outcome level two, but $92.51 \%$ of patients were discharged on an outcome level greater than two.

Table 1: The process of rehabilitation of patients with stroke

\begin{tabular}{ll}
\hline Process of rehabilitation & $\mathbf{N} \%$ \\
\hline Health professionals seen by patients & $7(10.60)$ \\
Psychologist & $65(98.48)$ \\
Doctor & $66(100.00)$ \\
Nurse & $63(95.45)$ \\
Occupational therapist & $65(98.48)$ \\
Physiotherapist & $36(54.54)$ \\
Speech therapist & $64(96.96)$ \\
Social worker & $11(16.66)$ \\
Dietician & \\
Length of hospital stay (days; SD) & $51.62(17.47)$ \\
Stroke population & $49.79(19.85)$ \\
Left brain lesions & $52.98(16.54)$ \\
Right brain lesion & \\
Discharge destination with referral (n; \%) & \\
Home & $55(82.08)$ \\
Health care facility & $4(5.97)$ \\
WCRC OPD & $5(7.46)$ \\
Other & $3(4.47)$ \\
Rehabilitation outcome levels $\%$ & \\
admission; discharge) & $22.39(0.00)$ \\
Level 1 & $56.72(7.49)$ \\
Level 2 & $16.42(31.34)$ \\
Level 3 & $2.99(50.75)$ \\
Level 4 & $0.00(10.45)$ \\
Level 5 & \\
\hline & \\
\hline
\end{tabular}




\section{Graded functional ability of participants}

The results presented in table 2 indicate that at the discharge assessment period only $18.18 \%$ of the participants were fully independent with the majority $(81.82 \%)$ still needing assistance with at least one activity of daily living at discharge.
Mean overall scores, standard deviation and significance level of functional abilities

The mean Barthel Index scores on admission and discharge were 58.85 (s.d. 24.70) and 81.59 (s.d. 14.91) respectively. A significant statistical difference was found between the mean admission and discharge Barthel Index scores $(p<0.01)$ as determined by the Student $\mathrm{t}$-test $(\mathrm{t}=8.18)$.

Table 2: The functional ability of patients' with stroke according to the Barthel Index Score

\begin{tabular}{lll}
\hline Category & $\begin{array}{l}\text { Admission } \\
\mathbf{n}=\mathbf{7 0} \mathbf{( \% )}\end{array}$ & $\begin{array}{l}\text { Discharge } \\
\mathbf{n}=\mathbf{6 6} \mathbf{( \% )}\end{array}$ \\
\hline Dependent (0 -55) & $35(50.00)$ & $5(7.58)$ \\
Moderate Assistance $(60-80)$ & $21(30.00)$ & $27(40.91)$ \\
Minimal Assistance $(85-95)$ & $11(15.71)$ & $22(33.33)$ \\
Independent (100) & $3(4.29)$ & $12(18.18)$ \\
\hline
\end{tabular}

Independent execution of tasks as measured by the Barthel Index

It is noticeable (see figure 1) that on discharge the independence of tasks were the highest for items relating to grooming, bladder continence and bathing with $95.45 \%$, $93.93 \%$ and $92.42 \%$ of patients performing the respective tasks independently. Furthermore, it is noteworthy to mention that irrespective of the increase in percentage of independent execution of the items relating to mobility and stair climbing between the two intervals, the percentages of independence remained the lowest. The greatest increases in the independent execution of tasks between admission and discharge were found for bathing, dressing and toileting with $55.11 \%, 45.90 \%$ and $33.99 \%$ of additional patients who were not independent with the execution of the respective tasks on admission.
Factors influencing functional outcomes at discharge The results (Table 3) from the linear regression suggest that only functional ability on admission, as measured by the Barthel Index, was found to have a statistical significant association with the outcome score at discharge. Other factors that were tested, but were found not to be significantly associated with the Barthel Index score on admission included gender, age, length of hospital stay, cognitive impairment and abnormal tone. Since the outcome score on admission is a numeric variable, the Spearman's correlation co-efficient was calculated to identify the relationship between the outcome score on admission and discharge. Even though the correlation is significantly different from 0 (Spearman's $r=0.49 ; \mathrm{p}<0.01$ ), it was not large numerically, but accounted for almost $50 \%$ of the variance in functional outcome.

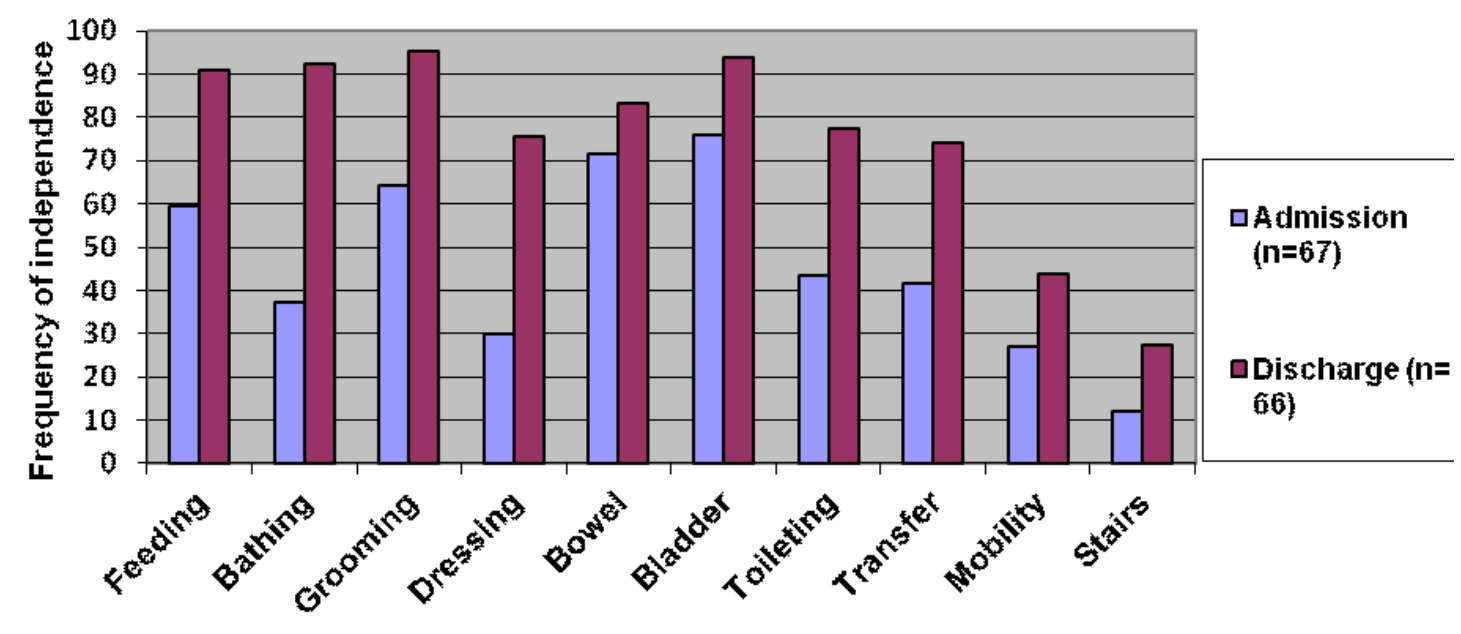

individual items

Figure 1: The independent execution of functional tasks on Barthel Index on admission and at discharge 
Table 3: Bivariate analysis of variables that could influence activity as measured by the Barthel Index at discharge $(n=66)$

\begin{tabular}{|c|c|c|c|c|c|}
\hline \multirow{2}{*}{$\begin{array}{l}\text { Variable } \\
\text { Gender }\end{array}$} & \multicolumn{2}{|c|}{$\begin{array}{l}\text { Nature of variable } \\
\text { Measurement }\end{array}$} & \multirow{2}{*}{$\begin{array}{l}\text { Test } \\
\text { Wilcoxon Rank }\end{array}$} & \multirow{2}{*}{$\begin{array}{l}\text { Spearman's } \\
\text { correlation } \\
\text { value } \\
\text { N/A }\end{array}$} & \multirow{2}{*}{$\begin{array}{l}\text { Significance } \\
\mathrm{p}=0.21\end{array}$} \\
\hline & Categorical & Male; Female & & & \\
\hline Age & Continuous & Years & Spearman's rho & $\mathrm{r}=(-) 0.17$ & $\mathrm{p}=0.16$ \\
\hline LOHS & Continuous & Days & Spearman's rho & $r=0.43$ & $\mathrm{p}=0.07$ \\
\hline BI (Admission) & Continuous & $0-100$ & Spearman's rho & $\mathrm{r}=0.49$ & $P<0.01$ \\
\hline Cognitive function & Categorical & Yes; No & Wilcoxon Rank & $\mathrm{N} / \mathrm{A}$ & $\mathrm{p}=0.21$ \\
\hline Abnormal tone & Categorical & 1-4 categories & Kruskal-Wallis & $\mathrm{N} / \mathrm{A}$ & $\mathrm{p}=0.75$ \\
\hline
\end{tabular}

LOHS-length of hospital stay; BI-Barthel Index on admission; N/A- Not applicable

\section{Discussion}

This study investigated activity limitations following in-patient rehabilitation and predictors of functional outcomes of patients with stroke in South Africa. The main findings indicate that the independent execution of activities of daily living as measured by the Barthel Index was greater in frequency for each item at discharge than on admission. However, stairs management, mobility, transfer and dressing were the activities in the present study with the lower frequency of independent execution at discharge. In addition, the results further emphasised the younger onset of stroke in the Western Cape, South Africa, which implicates that these survivors could have a longer life to live with the devastating consequences of stroke, if left untreated. Lastly, the study found that functional scores on admission was the only predictor of functional scores and abilities at discharge.

The inability of patients with stroke to perform activities of daily living such as dressing and transfer independently and safely, highlights the dependency patients with stroke, even those with mild impairment, have on others ${ }^{17}$. This functional dependence of patients is further reiterated by the results of this study demonstrating that less than $20 \%$ of the patients with stroke are fully independent, leaving more than $80 \%$ of patients dependent on others for at least one activity. Even though almost half of the patients are still significantly affected by the disability, which could be understood as patients needing assistance with at least one activity of daily living, more than $80 \%$ of the patients were discharged home without any formal follow-up rehabilitation services or routine medical screening for complications. Therefore, the risk of long-term disability associated with restrictions in certain human experiences, and possible preventative complications, could become the challenges these patients experience post-stroke. The discharge process/ destinations of the majority of the patients in this cohort could be challenged on an operational level, in which the institution embraced the patient-oriented approach to rehabilitation. Fundamentally this approach could be best understood within the ICF model which shifts the focus from measuring the normative to assessments of unique individualspecific difficulties encountered (both personal and environmental) and what an individual may need to become fully active and integrated members of his/ her society ${ }^{1,5}$. Therefore, these patients who are experiencing disability in any sphere of functioning should be provided with the needed support to achieve their goals for rehabilitation and to optimise their functioning.

Despite the functional limitations, the overall functional scores between the two data points demonstrated a significant improvement. Thus, the detection of statistical and clinical change cautiously underscores the importance and role of rehabilitation in the lives of patients with stroke. It should be noted that the findings related to statistical and clinical significant change are similar when compared to studies conducted among patients with stroke locally ${ }^{7,9}$ and internationally ${ }^{18}$. What is however novel to the local context, compared to the international literature, is the younger age onset of stroke, thus implicating the longer life persons have to live with their disability and the increased burden on the family and state for financial support and health care services. The age onset of stroke in the study sample was 52.95 years, suggesting that the majority of the patients are still at an employable age ${ }^{19}$. Previous research confirms that stroke occurs approximately 15 years earlier in the developing than in the developed world ${ }^{20}$. Despite the younger onset of stroke in the developing countries, literature suggests 
that younger survivors achieve better outcomes compared to their older counterparts, especially in function-related tasks, but not to an extent of complete independence ${ }^{21}$. This finding underlines the importance of continuous medical-and rehabilitation services if optimal outcomes are to be achieved, even for younger survivors of stroke.

Having highlighted the activities of daily living still performed with assistance at discharge, the need for further rehabilitation, perhaps on an out-patient basis, is apparent and imperative for achieving higher levels of functioning, not only within the domain of activity, but also for participation. The ICF clearly underpins the interrelationship between activity and participation, therefore the systematic approach of addressing activity limitations could culminate into more desirable functioning of patients within the dimensions of participation, which in return would give rise to an improved quality of life and satisfaction with life ${ }^{1,22}$. A recent study conducted among community dwelling stroke survivors found functional ability and independence to be the strongest predictor of, and were responsible for the greatest variance on participation $^{22}$.

For determining the factors influencing outcome with the use of bivariate testing, only the functional status on admission, as measured by the Barthel Index, had been significantly associated with the functional status at discharge. A direct relationship exists between activity scores, which suggest that the higher the functional score on admission the greater the score at discharge. This prediction corroborates with findings from other studies ${ }^{18,23}$. A study by Hoffmann and colleagues found that apart from the predictive nature of activity score (using the Barthel Index) on admission, it further had the greatest influence on social reintegration, which is often considered the desired outcome of rehabilitation ${ }^{24}$. Given the context of the current study, which clearly highlights the younger age onset of stroke with its own implications on prolonged dependency, rehabilitation should be geared towards systematically addressing the activity limitation within the context of the social environment of patients for not only improve tasks or actions as individual entities, but to improve social reintegration.

In this study, patient-related characteristics such as age and gender among those with stroke were found to be statistically insignificant on bivariate analysis. It was particularly surprising that age was not found to be a significant factor, as a negative association between age and functional outcomes is often reported in literature ${ }^{25}$. This could be as the stroke population at WCRC is unique and the profile differs from other populations worldwide. Not only were the mean ages in the sample lower than in overseas European countries, but age was seen as normally distributed, when one would expect it to be skewed. Only $16 \%$ of the patients with stroke were aged above 65 years. It could be argued that the negative manifestation of age is more evident with increasing age, which is often related to an increase in co-morbidities and where normal aging is more apparent and responsible for reduced functional ability and not the onset of the stroke per se.

No clear effect was found with regards to length of hospital stay, income, impairment variables and inherent factors such as age and gender. The small sample size reduces the power of the analyses ${ }^{16}$. However, the bivariate analysis gave insight into the factor that was found to influence functional outcome at discharge. The assessment of functional abilities on admission is essential for planning of the appropriate health professionals that should be involved in the rehabilitation of the patient and the specific interventions that are necessary to optimise functional recovery. On the contrary, the results that demonstrated insignificant correlation between independent variables and the functional outcome score at discharge could be evidence to place less emphasis and spend less of the scarce resources on the strategies that are implemented to address those variables. Also, the results should be taken with cautions as the study presented with a few limitations. Firstly, the convenient sampling strategy, with the exclusion of the patients with an advanced degree of receptive aphasia lead to results that cannot be extrapolated to all stroke survivors managed in practice. Secondly, data were also only collected from one rehabilitation setting, thus generalisability of these findings might be limited. Lastly, this particular paper underutilised the potential of the ICF as conceptual model and failed to investigate the interrelationship between domains and the influence of the contextual factors of the patient on outcome. Based on the limitations of this paper the authors recommend studies including larger samples sizes, studies with control groups (younger versus older stroke patients and rehabilitation versus non-rehabilitation) and studies investigating the contribution of the context, both personal and environmental, on the outcome of persons with stroke in order to facilitate comparison across countries.

African Health Sciences Vol 13 Issue 3 September 2013 


\section{Conclusion}

This study demonstrates the functional abilities and limitations of a predominantly younger cohort of stroke survivors following specialised in-patient rehabilitation. Despite the professional services available, patients are still discharged with apparent disability. In addition, this study highlights the need for the routine use of the Barthel Index, or any other relevant functional rating scale, with the purpose of setting functional goals for the patients and to identify the interventions necessary to optimise functional recovery. The implications of this study are twofold; firstly, the findings from this study could be used to adapt the existing rehabilitation programme to better target the essential activities of daily living of younger stroke survivors as derived from the outcome measures used, and secondly provides the basis for evaluating the content-, intensity- and appropriateness of interventions used to treat the activities still performed with greatest limitations.

\section{Acknowledgements}

The authors acknowledge the SANPAD reference group based at Stellenbosch for the financial assistance to complete this project. Furthermore, no conflict of interest is apparent in the conceptualisation, writing and submission of this article.

\section{References}

1. World Health Organisation. International Classification of Functioning, Disability and Health (ICF) 2001. Short version. Geneva, WHO.

2. Connor M, Bryer A. Stroke in South African. Chronic diseases of lifestyle in South Africa: 19952005. Technical Report 2006. Steyn, K, Fourie, F., \& Temple, N. Cape Town, South Africa. South African Medical Research Council.

3. World Health Organization. The Atlas of Heart Diseases and Stroke 2004a. Retrieved November 2, 2011, from http://apps.who.int/bookorders / MDIbooksPDF/Book/11500601.pdf

4. Aström M, Asplund K, Aström T. Psychosocial function and life satisfaction after stroke. Journal of the American Heart Association. 1992; 23: 527 531.

5. Loeb ME, Eide AH, Mont D. Approaching the measurement of disability prevelance: The case of Zambia. European Journal of Disability Research. 2008; 2: 32-43.

6. Hoenig H, Horner RD, Duncan PW, Clipp E, Hamilton B. New horizons in stroke rehabilitation research. Journal of Rehabilitation Research and Development. 1999; 36(1): 19-31.
7. Rhoda A, Mpofu R, De Weerdt W. Activity limitations of patients with stroke attending outpatient facilities in the Western Cape, South Africa. South African Journal of Physiotherapy. 2011; 67(2): 16-22.

8. Urimubenshi G, Rhoda A. Process of Physiotherapy services of stroke patients treated at Rhuhengeri Hospital in Rwanda: A four year document review. Journal of Community and Health Sciences. 2010; 5(1): 26-33.

9. Rouillard S. Pattern of recovery and outcome after stroke in patients accessing a Western Cape Rehabilitation facility. Unpublished Masters 2006. University of Cape Town, Cape Town.

10. Macko RF, Ivey FM, Forrester LW. Treadmill exercise rehabilitation improves ambulatory function and cardiovascular fitness in patients with chronic stroke: a randomized, controlled trial. Stroke. 2005; 36: 2206-2211.

11. Scheepers VPM, Ketelaar M, Van De Port IGL, Visser-Meily JMA, Lindeman E. Comparing contents of functional outcome measures in stroke rehabilitation using the International Classification of Functioning, Disability and Health. Disability and Rehabilitation. 2007; 29(3): 221-230.

12. Kaplan SL. Outcome measurement and Management: First Steps for the Practicing Clinician. United States of America: F.A. Davis Company, 2007.

13. Scivoletto G, Morganti B, Ditunno P, Ditunno JF, Molinari M. Effects on age on spinal cord lesion patients' rehabilitation. Spinal Cord. 2003; 41(8): 457-464.

14. Wade DT, Hewer RL. Functional abilities after stroke: measurement, natural history and prognosis. Journal of Neurology, Neurosurgery, Psychiatry. 1987; 50: 177-182.

15. Mahorney FI, Barthel DW. Functional evaluation: the Barthel Index. Maryland State Medical Journal. 1965;14: 56-61.

16. Domholdt E. Physical Therapy Research: Principles and Application (2 $2^{\text {nd }}$ ed.). Philadelphia: WB Saunders Publishers, 2000.

17. Dowswell G, Lawler J, Dowswell T, Young J, Forster A, Hearn J. Investigating recovery from stroke: a qualitative study. Journal of Clinical Nursing. 2000; 9(4): 507-515.

18. Kuptniratsaikul V, Kovindha A, Dajpratham P, Piravej K. Main outcomes of stroke rehabilitation: A multi-centre study in Thailand. Journal of Rehabilitation Medicine. 2009; 41: 54-58. 
19. Statistics South Africa. Provincial Profile 2004: Western Cape. Pretoria, Statistics South Africa, 2006.

20. Bonita R, Mendis S, Truelsen T, Bogousslavsky J, Toole J, Yatsu F. The global stroke initiative. Lancet Neurology. 2004; 3: 391-393.

21. Kalra L. Does age affects benefits of stroke unit rehabilitation? Stroke. 1994; 25: 346-351.

22. Chau JPC, Thompson DR, Twinn S, Chang AM, Woo J. Determinants of participation restriction among community dwelling stroke survivors: A path analysis. Bio Med Central Neurology. 2009; 9(49): 1-7.
23. Duncan PW, Zorowitz R, Bates B, Choi JY, Glasberg JJ, Graham GD, et al. Management of adult stroke rehabilitation care: A clinical practice guideline. Stroke. 2005; 36: e100-e143.

24. Hoffmann T, McKenna K, Cooke D, Tooth L. Outcomes after stroke: Basic and instrumental activities of daily living, community reintegration and generic health status. Australian Occupational Therapy Journal. 2003; 50: 225-233.

25. Kwakkel G, Wagenaar RC, Kollen BJ, Lankhorst GJ. Predicting disability in stroke-a critical review of the literature. Age aging. 1996; 25(6): 479-489. 